tant in maintaining ISCs, in a manner analogous to GSCs. In any case, stem cells such as ISCs, which sense local cellular requirements and produce appropriate daughter cells in response, likely play a central role in the physiology, longevity, and pathology of the tissues they maintain.

\section{References and Notes}

1. T. Nystul, A. C. Spradling, Curr. Opin. Genet. Dev. 16 , 463 (2006).

2. D. T. Scadden, Nature 441, 1075 (2006).

3. T. Xie, A. C. Spradling, Cell 94, 251 (1998).

4. T. Xie, A. C. Spradling, Science 290, 328 (2000).

5. X. Song, T. Xie, Development 130, 3259 (2003).

6. N. Tulina, E. Matunis, Science 294, 2546 (2001).
7. A. Kiger, D. L. Jones, C. Schulz, M. B. Rogers, M. T. Fuller, Science 294, 2542 (2001).

8. S. L. Crittenden, K. A. Leonhard, D. T. Byrd, ]. Kimble, Mol. Biol. Cell 17, 3051 (2006).

9. L. M. Calvi et al., Nature 425, 841 (2003).

10. J. Zhang et al., Nature 425,836 (2003).

11. B. Ohlstein, A. C. Spradling, Nature 439, 470 (2006).

12. C. Michelli, N. Perrimon, Nature 439, 475 (2006).

13. See supporting material on Science Online.

14. H. Jafar-Nejad et al., Dev. Cell 9, 351 (2005).

15. S. Bray, Nat. Rev. Mol. Cell Biol. 7, 678 (2006).

16. M. Furriols, S. Bray, Curr. Biol. 11, 60 (2001).

17. P. Heitzler, P. Simpson, Cell 64, 1083 (1991).

18. E. Pavlopoulos et al., Dev. Cell 1, 807 (2001)

19. R. Le Borgne, S. Remaud, S. Hamel, F. Schweisguth, PLoS Biol. 3, e96 (2005).
20. M. Bjerknes, H. Cheng, Am. J. Physiol. 289, G381 (2005)

21. R. Roegiers, Y. N. Jan, Curr. Opin. Cell Biol. 16, 195 (2004).

22. A. Gromley et al., Cell 123, 75 (2005).

23. We thank M. Buszczak and T. Nystul for comments on the manuscript and H. Ruhoula-Baker, K. Irvine, C. Field, S. DiNardo, and I. Rebay for gifts of materials.

\section{Supporting Online Material}

www.sciencemag.org/cgi/content/full/315/5814/988/DC1

Materials and Methods

Fig. S1

References

23 October 2006; accepted 8 January 2007

10.1126/science.1136606

\title{
Polymerizing Actin Fibers Position Integrins Primed to Probe for Adhesion Sites
}

\section{Catherine G. Galbraith, ${ }^{1}$ Kenneth M. Yamada, ${ }^{1 *}$ James A. Galbraith ${ }^{2}$}

Migrating cells extend protrusions, probing the surrounding matrix in search of permissive sites to form adhesions. We found that actin fibers polymerizing along the leading edge directed local protrusions and drove synchronous sideways movement of $\beta_{1}$ integrin adhesion receptors. These movements lead to the clustering and positioning of conformationally activated, but unligated, $\beta_{1}$ integrins along the leading edge of fibroblast lamellae and growth cone filopodia. Thus, rapid actin-based movement of primed integrins along the leading edge suggests a "sticky fingers" mechanism to probe for new adhesion sites and to direct migration.

$\mathrm{T}$ he first steps of mammalian cell migration are extension of the leading edge and attachment to the surrounding extracellular matrix (1). Extension is driven by actin polymerization (2), with the position (3), speed, and persistence (4) of the leading edge regulated by actin-binding proteins. However, extension is not uniform; adjacent regions of many broad, flat protrusions such as fibroblast lamellae and neuronal growth cones advance and retract independently, and which region moves forward is continually redefined by transverse movement $(5,6)$. Observations on protrusion dynamics led to the hypothesis that these protrusion variations are used to search for permissive sites to form new adhesions $(7,8)$. However, no known direct relation between protrusion dynamics and the ability of adhesion receptors to probe the matrix has been identified.

We studied protrusion dynamics during the initial matrix exploration. Using high-contrast differential interference contrast (DIC) optics, we observed small ripple protrusions traversing the outermost edge of fibroblast lamellipodia, the leading 1 to $2 \mu \mathrm{m}$ of the lamella (Fig. 1, A and B). The ripples did not move sideways at the

${ }^{1}$ National Institute of Dental and Craniofacial Research, National Institutes of Health, Bethesda, MD 20892, USA ${ }^{2}$ National Institute of Neurological Disorders and Stroke, National Institutes of Health, Bethesda, MD 20892, USA.

*To whom correspondence should be addressed. E-mail: kyamada@dir.nidcr.nih.gov same speed $(0.10 \pm 0.06 \mu \mathrm{m} / \mathrm{s}, n=34)$ that actin network treadmilling $(0.028 \pm 0.013 \mu \mathrm{m} / \mathrm{s}, n=$ 51) (9) moved microspikes and filopodia precursors laterally (Fig. 1D) $(10,11)$. Instead, the ripples moved at the same speed that actin polymerization elongates growth cone filopodia (12) and rockets Listeria bacteria (13). Although the frequency of ripples decreased in response to the actin-depolymerizing agent cytochalasin D (Fig. 1C), the associated actin dynamics are difficult to visualize with green fluorescent protein (GFP)-actin because of the density and rapid turnover (9). We therefore exploited the properties of photoactivatable GFP (PaGFP) (14) to establish the threshold of visible actin and to restrict visualization to $\sim 40 \%$ of the expressed PaGFP-actin (15). Limiting activation revealed localized increases in actin intensity that moved sideways with the ripples (Fig. 1D and movie S1). The increases did not represent thickness variations or membrane lifting, because they persisted after normalization by the cotransfected monomeric red fluorescent protein (mRFP) signal, and the ripples remained within a single DIC optical section (15). The intensity increases are local actin polymerization at crests of ripples traversing the leading edge ( $82 \%$ colocalization, $n=198)$ (Fig. 1D and movie S1) (15).

Restricting the region of photoactivation revealed that activated PaGFP-actin localized at the tip of a nascent ripple and accumulated along the elongating ripple (Fig. 2A). In contrast to this
Fig. 1. Local increases in actin polymerization occur at small ripples traversing the leading edge of migratory fibroblasts. (A) DIC and PaGFP-actin images showing NIH3T3 fibroblast lamella (l) and lamellipodium (lp). Region of cell is shown in inset, and black box indicates region in (D). Scale bar, $5 \mu \mathrm{m}$. (B) Histogram of the ripple duration or wave period ( $n=106$, 21 cells) (15). Kymographs from DIC images show the duration of the transverse ripples (white bracket) and local forward protrusions (black bracket). Horizontal scale bar, $20 \mathrm{~s}$; vertical, $0.5 \mu \mathrm{m}$. (C) Cytochalasin D (CD) decreases the frequency of the ripples. (D) At the tips of ripples, there are localized increases in PaGFP-actin fluorescent intensity (red arrowheads). The ripples move sideways faster than either the lateral movement of filopodia (vertical yellow arrowheads) or the rearward movement of actin (horizontal yellow arrowheads) due to lamella network treadmilling. Dashed lines mark initial positions of yellow arrowheads. Scale bar, $5 \mu \mathrm{m}$.
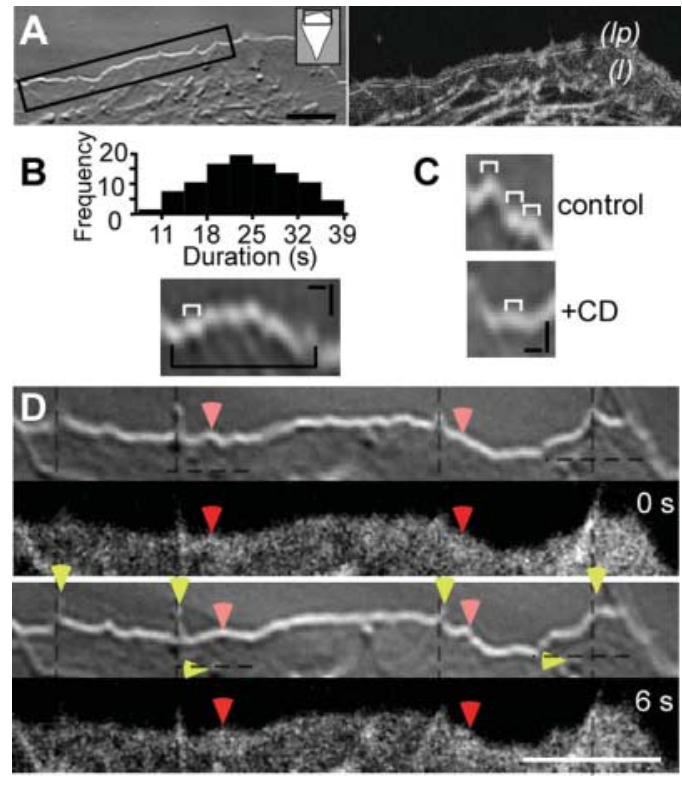
asymmetric accumulation of PaGFP-actin, photoactivation of PaGFP alone produced a symmetric signal that spread throughout the cell and was consistent with simple diffusion (fig. S1). We also found that the vasodilator-stimulated phosphoprotein (VASP), which protects barbed ends from capping (4), localized to actin elongating across the leading edge (Fig. 2B and movie S2). The elongating VASP-tipped actin reshaped and redirected the larger regions of forward protrusion (movie S2). Thus, it seems that the transverse ripples represent local sites of prefer- ential actin elongation along the edge of advancing protrusions.

To test if the localized increases in actin polymerization are involved in matrix probing, we compared them with the location of integrin adhesion receptors. Using a fluorescent, nonperturbing antibody, we observed clustering of $\beta_{1}$ integrins that was temporally and spatially synchronized with actin polymerization at the crests of ripples (Fig. 2, D to F, and movie S2). The $\beta_{1}$ integrins and actin remained colocalized during an $\sim 3$-min monitoring period (correlation
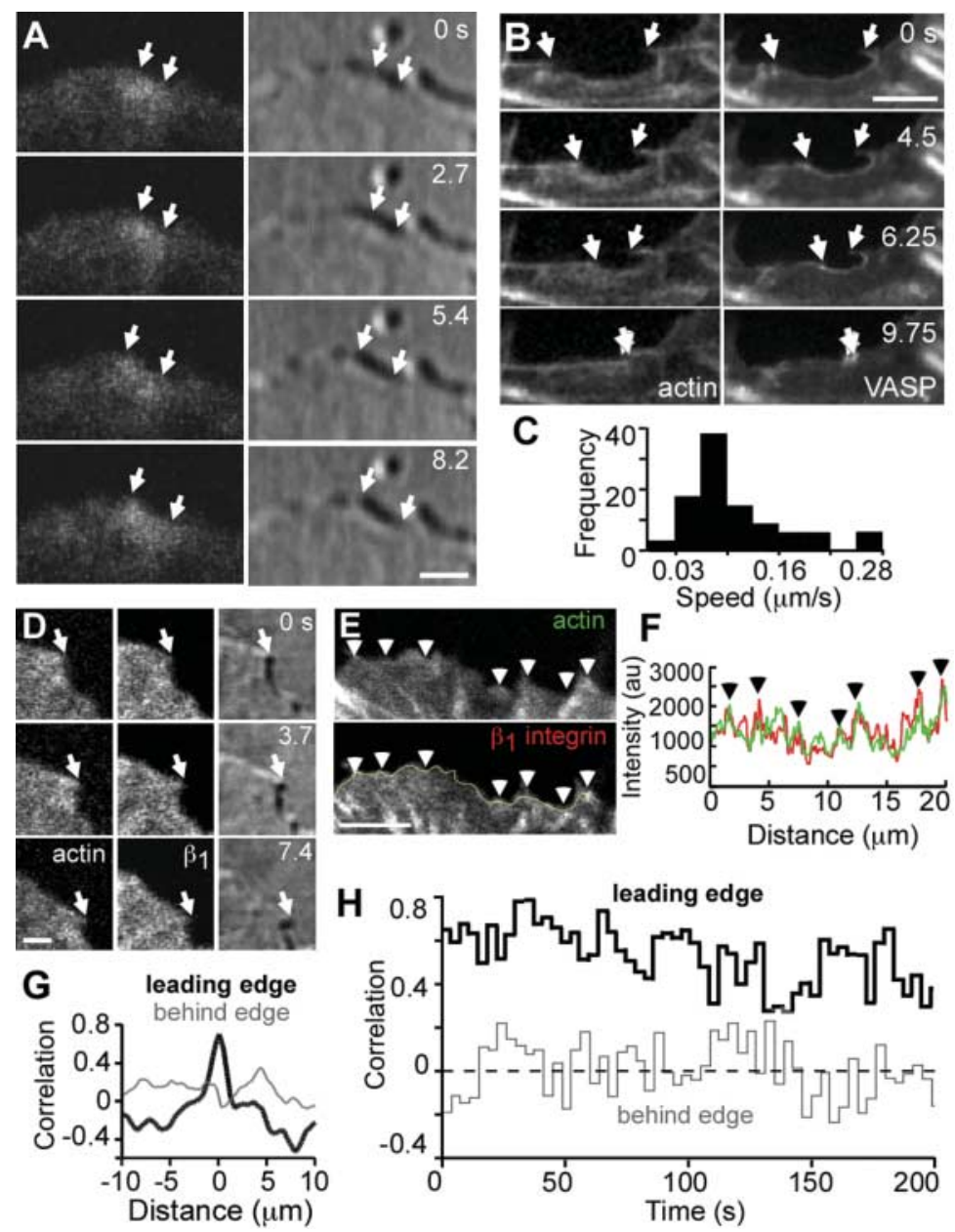

Fig. 2. $\beta_{1}$ Integrins move synchronously with actin polymerizing at the crests of ripples. (A) Activated PaGFP-actin (fluorescence) initially localizes to the tip of a nascent ripple (DIC) and accumulates along the entire ripple as it elongates. Arrows slanted toward direction of ripple movement. Scale bar, $1 \mu \mathrm{m}$. (B) VASP, which enhances actin polymerization (4), is localized at the tips of actin fibers elongating along the leading edge. Scale bar, $5 \mu \mathrm{m}$. (C) Histogram of ripple speed ( $n=34,27$ cells). (D) A sequence from movie S3 shows enhanced green fluorescent protein (EGFP)-actin and $\beta_{1}$ integrin moving synchronously with the tip of an elongating ripple (arrows). Scale bar, $1 \mu \mathrm{m}$. (E) The first image of movie S3 shows EGFP-actin and $\beta_{1}$ integrins have corresponding increases in intensity. The yellow contour indicates the single-pixel-wide line used to obtain the intensity traces shown in (F). Scale bar, $5 \mu \mathrm{m}$. (F) Fluorescent intensity of EGFP-actin (red) and $\beta_{1}$ integrin (green) for image in (E); arrowheads correspond to those in (E). (G) Crosscorrelation analysis shows a strong correlation between the intensity traces of EGFP-actin and $\beta_{1}$ integrin at the edge and essentially no correlation behind the edge. (H) A high correlation between EGFP-actin and $\beta_{1}$ integrins is maintained along a $20 \mu \mathrm{m}$ length of the leading edge throughout the $\sim 3$-min monitoring period (correlation coefficient $0.53 \pm 0.13$ at the leading edge, $n=54$, and $0.01 \pm 0.14$ behind the lamellipodia in a region devoid of adhesive contacts, $n=54$ ). (A to $C$ ) are NIH3T3 cells and (D to $H$ ) are human foreskin fibroblasts. coefficient $0.50 \pm 0.12, n=217$, five cells). In contrast, $\beta_{1}$ integrins did not colocalize with actin behind the leading edge (correlation coefficient $0.01 \pm 0.16, n=140$, five cells) (Fig. 2, G and H, and movie S3) (15). Similar correlations were obtained for positive and negative controls, respectively (fig. S3). Thus, $\beta_{1}$ integrins move synchronously with polymerizing actin along the leading edge.

To test whether these $\beta_{1}$ integrins were capable of binding extracellular matrix, we examined their conformational state and found activated $\beta_{1}$ integrins colocalized to the tips of actin along the leading edge (correlation coefficient $0.63 \pm 0.10$, $n=14$ ) (Fig. 3, A and B, figs. S2 to S5). To visualize activation and to determine where the transition to a high-affinity state occurred, we developed a live-cell assay using a fluorescent antibody targeting high-affinity $\beta_{1}$ integrins (9EG7). We initially labeled all of the activated $\beta_{1}$ integrins on the cell surface so that any subsequent labeling would reveal newly activated $\beta_{1}$ integrins. These newly labeled integrins appeared only at the leading edge (Fig. 3C and movie S4).

The activated $\beta_{1}$ integrins were not ligated to fibronectin but could bind fibronectin fragments (FN120kD), specifically, with the $\alpha_{5} \beta_{1}$ integrin heterodimer (Fig. 3D and fig. S2), which was consistent with an increased avidity for fibronectin at the leading edge $(16,17)$. Although unligated high-affinity $\alpha_{v} \beta_{3}$ integrins, which can bind vitronectin or fibronectin, transiently localize to the edge of spreading cells (18), we did not find $\beta_{3}$ integrin localization (fig. S3) or increased vitronectin avidity at the leading edge of migrating cells $(17,19)$. Moreover, the observed spatial correlation between high-affinity $\beta_{1}$ integrins and actin was not restricted to a single cell type, antibody, or extracellular matrix (Fig. 4 and fig. S3). Activated, but unligated, fibronectin adhesion receptors at the leading edge may thus have a direct role in sampling the extracellular matrix during migration.

We tested the functional relation between actin polymerization and activated $\beta_{1}$ integrins by altering actin dynamics (Fig. 4A and fig. S4). Decreasing the number of polymerizing actin ends by capping with cytochalasin $\mathrm{D}$ decreased the density of activated $\beta_{1}$ integrins, while increasing the number of polymerizing ends with the stabilizer jasplakinolide ( 9 ) increased the activated $\beta_{1}$ integrin density at the cell periphery. Moreover, increasing actin turnover without changing the number of barbed ends, with the myosin II adenosine triphosphatase (ATPase) inhibitor blebbistatin, $(15,20)$ did not change the activated $\beta_{1}$ integrin density. Consistent changes in activated $\beta_{1}$ integrin density were observed with siRNA (small interfering RNA) knockdown of the cofilin phosphatase slingshot and capping protein $\beta_{2}$ (fig. S4). Despite the changes in density, the correlation between activated $\beta_{1}$ integrins and polymerizing actin remained high [correlation coefficient 0.49 to 0.54 with no statistical difference $(P>0.05)$ 
Fig. 3. Activated but unligated $\beta_{1}$ integrins are located at the tips of actin fibers at the leading edge. (A) Conformationally activated $\beta_{1}$ integrins are spatially concentrated at the tips of polymerizing actin at the leading edge of human foreskin fibroblasts. Scale bar, $5 \mu \mathrm{m}$. (B) Fluorescent intensity of F-actin (red), activated $\beta_{1}$ integrin (blue), and total (active + inactive) $\beta_{1}$ integrin (green) along the yellow line in (A). In this example, the correlation coefficient is 0.69 between total $\beta_{1}$ integrin and F-actin, and 0.75 between activated $\beta_{1}$ integrin and F-actin. The average correlation coefficients for multiple cells are shown in fig. S3 and Fig. 2D $(n=14)$. Total and active integrin distributions are compared in fig. S2D. Scale bar, $5 \mu \mathrm{m}$. (C) Sequential fluorescent (top) and DIC (bottom) images showing the activation of $\beta_{1}$ integrins on NIH3T3 cells. Different color arrowheads differentiate individual activation-specific antibody binding sites at the leading edge that

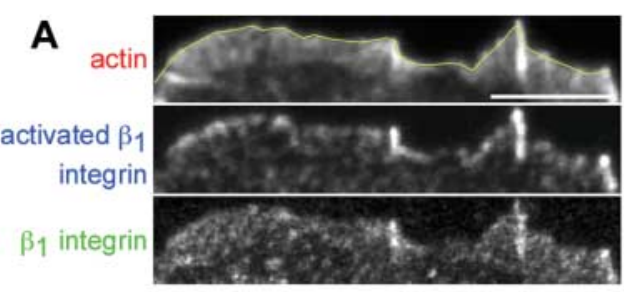

B

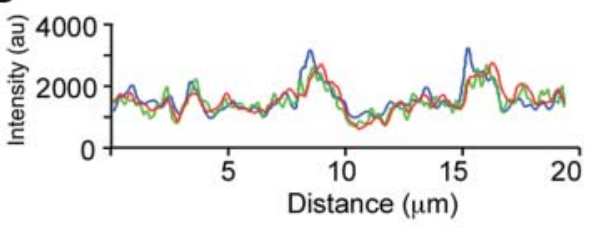

C
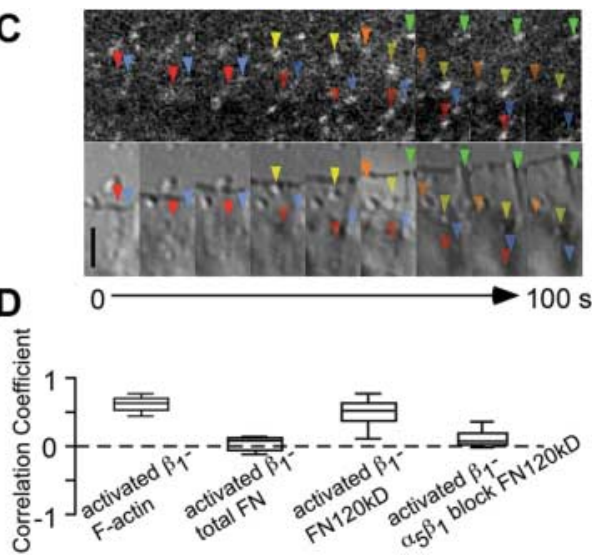

Fig. 4. Localization of activated $\beta_{1}$ integrins at the leading edge depends on availability of polymerizing actin ends. (A) NIH3T3 cells stained for activated $\beta_{1}$ integrins (green) and F-actin (red and grayscale) show a decrease in activated $\beta_{1}$ integrin density when actin is capped (cytochalasin D, $n=19$ ) and an increase when actin is stabilized to increase the number of free ends (jasplakinolide, $n=22$ ). Inhibiting myosin II ATPase (blebbistatin, $n=18$ ) did not change the number of actin ends or the integrin concentration. Scale bar, $5 \mu \mathrm{m}$. Boxplot of activated $\beta_{1}$ integrin density normalized to control $(n=27)$. (B) Activated $\beta_{1}$ integrins are also localized to the tips of polymerizing growth cone filopodia (NG108 cells). Scale bar, $5 \mu \mathrm{m}$.
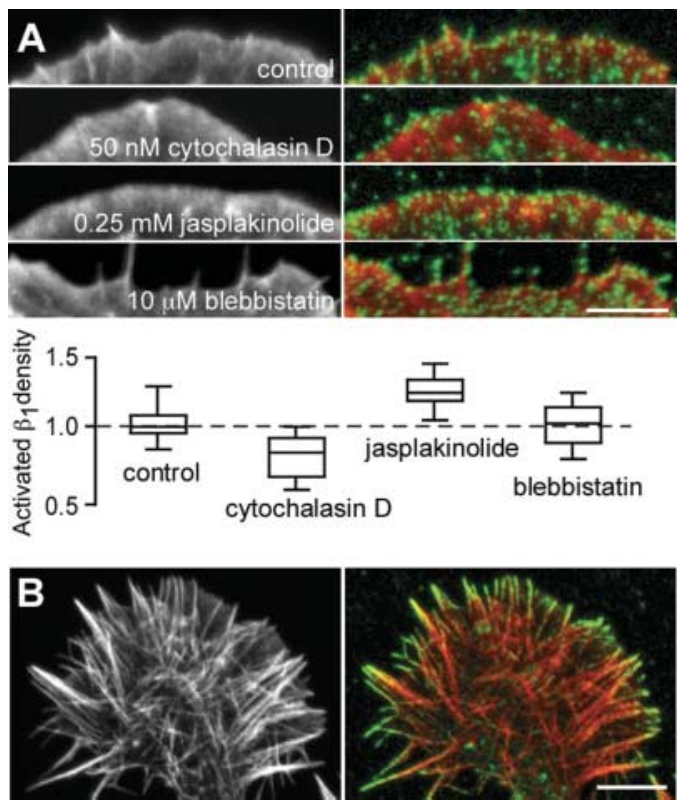

between cytochalasin $\mathrm{D}(n=23)$, jasplakinolide $(n=27)$, blebbistatin $(n=34)$, and control $(n=$ 25)] (15). The changes in activated $\beta_{1}$ integrin density thus correspond to the number of polymerizing actin ends.

To further test whether $\beta_{1}$ integrins interact with polymerizing actin, we demembranated cells with Triton X-100 to remove the cell membrane and membrane-associated proteins but leave behind the cytoskeleton and cytoskeletally associated proteins (15). Activated $\beta_{1}$ integrins quantitatively colocalized with the tips of polymerizing actin at the leading edge in the demembranated cells (fig. S5A). The leading edge localization of activated and total $\beta_{1}$ integrins was decreased by siRNA knockdown of talin 1 , a direct physical linker between actin and $\beta_{1}$ integrins (21) that is associated with tips of actin fibers along the leading edge (22). Ad- ditionally, the localization was not changed by knockdown of $\alpha$-actinin 1 , another direct linker (23) that is not similarly concentrated along the leading edge (fig. S5C). These data suggest that talin contributes to the linkage between $\beta_{1}$ integrins and polymerizing actin at the leading edge.

We also found activated $\beta_{1}$ integrins localized at the tips of growth cone filopodia (Fig. 4B). Growth cones guide migration by sampling the matrix for ligands and relative stiffness (24), and their actin-based filopodia are necessary for correct steering in vivo (25). The localization of activated $\beta_{1}$ integrins at filopodia tips suggests a role in growth cone path-finding. Additionally, we tested whether the activated adhesion receptors at the cell perimeter regulated migration force to match matrix stiffness by using an optical trap assay $(17,24,26)$. We found connections formed between fibronectin and the adhesion receptors at ripple protrusions were more likely to regulate migration force $(85 \%$, $n=13$ ) than connections formed at other regions along the leading edge $(25 \%, n=12)$. This suggests that activated $\beta_{1}$ integrins are localized at the ends of actin fibers to probe for ligands, to guide migration direction, and to regulate migration force.

Polymerization of actin fibers directs the movement of a variety of protrusions ranging from rocketing Listeria (27) to elongating filopodia (12). Our data indicate that preferential localized polymerization and elongation of actin filaments or fibers along the leading edge steer local forward protrusions. The polymerizing actin moved and clustered $\beta_{1}$ integrins to spatially position integrins primed to probe the matrix at the very front of cell protrusions, creating "sticky fingers" along the leading edge. Transverse variations in actin polymerization along the advancing perimeter allow the primed integrin receptors to sample more of the extracellular matrix than simple forward and backward movement. This might allow cells with well-developed adhesive contacts to make small changes in migration direction without severing and reestablishing their matrix connections under the cell body. Thus the extremely dynamic local variations in actin polymerization probe the matrix for adhesion sites by positioning primed adhesion receptors along the advancing cell perimeter during migration.

\section{References and Notes}

1. A. J. Ridley et al., Science 302, 1704 (2003).

2. T. D. Pollard, G. G. Borisy, Cell 112, 453 (2003).

3. M. Ghosh et al., Science 304, 743 (2004).

4. J. E. Bear et al., Cell 109, 509 (2002).

5. M. Abercrombie, J. E. Heaysman, S. M. Pegrum, Exp. Cell Res. 59, 393 (1970).

6. M. Machacek, G. Danuser, Biophys. J. 90, 1439 (2006).

7. M. Abercrombie, ]. E. Heaysman, S. M. Pegrum, Exp. Cell Res. 62, 389 (1970). 
8. G. Giannone et al., Cell 116, 431 (2004).

9. N. Watanabe, T. J. Mitchison, Science 295, 1083 (2002).

10. G. Danuser, R. Oldenbourg, Biophys. J. 79, 191 (2000).

11. T. M. Svitkina et al., J. Cell Biol. 160, 409 (2003).

12. A. Mallavarapu, T. Mitchison, J. Cell Biol. 146, 1097 (1999).

13. J. A. Theriot, T. J. Mitchison, L. G. Tilney, D. A. Portnoy, Nature 357, 257 (1992).

14. G. H. Patterson, ]. Lippincott-Schwartz, Science 297 1873 (2002)

15. Materials and methods are available as supporting material on Science Online.

16. T. Nishizaka, Q. Shi, M. P. Sheetz, Proc. Natl. Acad. Sci. U.S.A. 97, 692 (2000).

17. C. G. Galbraith, K. M. Yamada, M. P. Sheetz, J. Cell Biol. 159, 695 (2002)

18. W. B. Kiosses, S. J. Shattil, N. Pampori, M. A. Schwartz, Nat. Cell Biol. 3, 316 (2001).

19. D. P. Felsenfeld, P. L. Schwartzberg, A. Venegas, R. Tse, M. P. Sheetz, Nat. Cell Biol. 1, 200 (1999).
20. N. A. Medeiros, D. T. Burnette, P. Forscher, Nat. Cell Biol. 8, 215 (2006)

21. A. Horwitz, K. Duggan, C. Buck, M. C. Beckerle, K. Burridge, Nature 320, 531 (1986).

22. ]. A. DePasquale, C. S. Izzard, J. Cell Biol. 113, 1351 (1991).

23. C. A. Otey, F. M. Pavalko, K. Burridge, ]. Cell Biol. 111 , 721 (1990).

24. D. M. Suter, P. Forscher, J. Cell Biol. 155, 427 (2001).

25. D. Bentley, A. Toroian-Raymond, Nature 323, 712 (1986).

26. D. Choquet, D. P. Felsenfeld, M. P. Sheetz, Cell 88, 39 (1997).

27. T. P. Loisel, R. Boujemaa, D. Pantaloni, M. F. Carlier, Nature 401, 613 (1999).

28. We thank C. Smith and T. Reese for suggestions on the experiments and manuscript and $M$. Sheetz for use of optical trap data collected by C.G.G. while in his laboratory. For use of the laser scanning microscopes, we thank the National Institute of Neurological Disorders and Stroke (NINDS) Light
Imaging Facility for the LSM 510 (VIS/405) and Carl Zeiss, Inc., for the LSM 5 LIVE and LSM 5 DUO. We also thank Z. Iwinski for confocal software; E. Shumsky,

R. Engelmann, and S. Tille for assistance; $G$. Patterson and ]. Lippincott-Schwartz for PaGFP and discussions; and $M$. Hoffman for assistance with reverse transcriptionpolymerase chain reaction studies. Reagents provided by R. Tsien (mRFP), ]. Wehland (EGFP-VASP), and S. Yamada (human foreskin fibroblasts). Support provided by the intramural research programs of NINDS and National Institute of Dental and Craniofacial Research, NIH.

Supporting Online Material

www.sciencemag.org/cgi/content/full/315/5814/992/DC1 Materials and Methods

Figs. $\mathrm{S} 1$ to $\mathrm{S} 5$

References

Movies S1 to S4

2 November 2006; accepted 5 January 2007

$10.1126 /$ science. 1137904

\section{Maplike Representation of Celestial E-Vector Orientations in the Brain of an Insect}

\section{Stanley Heinze and Uwe Homberg*}

For many insects, the polarization pattern of the blue sky serves as a compass cue for spatial navigation. $E$-vector orientations are detected by photoreceptors in a dorsal rim area of the eye. Polarized-light signals from both eyes are finally integrated in the central complex, a brain area consisting of two subunits, the protocerebral bridge and the central body. Here we show that a topographic representation of zenithal $E$-vector orientations underlies the columnar organization of the protocerebral bridge in a locust. The maplike arrangement is highly suited to signal head orientation under the open sky.

$\mathrm{M}$ any animals, including birds, fishes, cephalopods, and arthropods, share the ability to perceive linearly polarized light $(1,2)$. The plane of polarization ( $E$-vector) varies systematically across the blue sky and depends on the Sun's position. For a variety of insects this pattern has been shown to guide spatial orientation (2). In locusts, polarotactic orientation depends on a specialized part of the compound eye, the dorsal rim area (3), and involves several central processing stages, including the central complex (4-7). The central complex (CC) is a group of neuropils spanning the midline of the insect brain. Substructures are the protocerebral bridge (PB) and the upper and lower divisions of the central body. An outstanding anatomical feature of the $\mathrm{CC}$ is its regular and highly sophisticated internal neuroarchitecture $(8-10)$. In simplified terms, it consists of stacks of arrays, each composed of a linear arrangement of 16 columns with topographic interhemispheric connections between columns both within and between different arrays. Hypotheses on the functional

Animal Physiology, Department of Biology, Philipps University, 35032 Marburg, Germany.

*To whom correspondence should be addressed. E-mail: homberg@staff.uni-marburg.de roles of the $\mathrm{CC}$ range from a control center for motor coordination (11) to a recently demonstrated involvement in visual pattern learning and recognition (12). In the locust, several cell types of the $\mathrm{CC}$ are sensitive to the orientation of zenithal $E$-vectors (5), but the correspondence of cell morphology and $E$-vector tuning has remained obscure. In this study, we have used intracellular recordings combined with dye injections to analyze $E$-vector tuning in CC neurons of the locust with columnar arborization domains.

Two major classes of polarization-sensitive (POL) neurons were encountered regularly when we recorded from CC neurons: (i) a particular type of tangential neuron of the $\mathrm{PB}$ and (ii) several types of columnar neurons. Tangential neurons of the PB, termed here TB1 neurons, have not been described previously in the locust or any other insect. A total number of 18 of these cells were analyzed. Their morphology was revealed by iontophoretic tracer injection, histological processing, and camera lucida reconstruction (13). TB1 neurons provide a connection between a posterior brain region, the posterior optic tubercle, and the PB (Fig. 1, A and B). Each TB1 neuron had two domains of varicose and, thus, putatively presynaptic arborizations con- fined to a single column in each hemisphere of the PB. When PB columns are numbered as L1 (lateralmost column in the left hemisphere of the PB) to L8 (most medial column in the left hemisphere) and, accordingly, from R1 to R8 in the right hemisphere of the bridge, TB1 neurons with varicose ramifications in columns $\mathrm{R} 1 / \mathrm{L} 8$, R2/L7, R4/L5, R5/L4, R6/L3, and R7/L2 were encountered. Varicose processes were always eight columns apart, with processes ipsilaterally in one of the four outer columns and contralaterally in one of the four inner columns (Fig. 1E). The columns neighboring those with varicose processes were free of ramifications, and six to eight other columns were invaded with fine, smooth arborizations. Furthermore, all TB1 neurons had varicose arborizations in the posterior optic tubercle, a brain area connected to a small neuropil in the optic lobe, the accessory medulla (14).

The pattern of varicose arborizations in the PB corresponded to physiological properties of the TB1 neurons. For intracellular recordings, the animals were fixed in the recording setup and stimulated from the zenith with a rotating $E$-vector. Recordings were obtained from their main neurite in the PB. Each TB1 neuron showed polarization opponency, i.e., $E$-vector orientations leading to an increase in spiking activity (excitation) were oriented perpendicularly to $E$-vectors, leading to a decrease in spiking activity (inhibition) (Fig. 1, C and D). The $E$-vector tuning ( $E$-vector orientation resulting in maximum excitation, $\Phi_{\max }$ ) was determined for each neuron by circular statistics [Rayleigh test (15)]. $E$-vector tuning of TB1 neurons showed a linear relation to the position of their varicose ramifications in the PB (Fig. 1F). Thereby, a range of $\Phi_{\max }$ tunings of $182^{\circ} \pm 71^{\circ}$ extends through the eight columns of each hemisphere of the PB and, thus, corresponds to the whole range of possibly occurring $E$-vector orientations.

Because the $E$-vector map in the PB corresponds with the proposed output regions of the TB1 neurons, we asked whether candidate postsynaptic neurons show a similar representation of $E$-vector tuning. Columnar neurons have ar- 